\title{
ON METRIC CONNECTIONS WITH TORSION ON THE COTANGENT BUNDLE WITH MODIFIED RIEMANNIAN EXTENSION
}

\author{
LOKMAN BILEN AND AYDIN GEZER
}

\begin{abstract}
Let $M$ be an $n$-dimensional differentiable manifold equipped with a torsion-free linear connection $\nabla$ and $T^{*} M$ its cotangent bundle. The present paper aims to study a metric connection $\widetilde{\nabla}$ with nonvanishing torsion on $T^{*} M$ with modified Riemannian extension $\bar{g}_{\nabla, c}$. First, we give a characterization of fibre-preserving projective vector fields on $\left(T^{*} M, \bar{g}_{\nabla, c}\right)$ with respect to the metric connection $\widetilde{\nabla}$. Secondly, we study conditions for $\left(T^{*} M, \bar{g}_{\nabla, c}\right)$ to be semi-symmetric, Ricci semi-symmetric, $\widetilde{Z}$ semi-symmetric or locally conharmonically flat with respect to the metric connection $\widetilde{\nabla}$. Finally, we present some results concerning the Schouten-Van Kampen connection associated to the Levi-Civita connection $\bar{\nabla}$ of the modified Riemannian extension $\bar{g}_{\nabla, c}$.

Mathematics subject classification 2010. 53C07, 53C35, 53A45.

Key words and phrases. Cotangent bundle, fibre-preserving projective vector field, metric connection, Riemannian extension, semi-symmetry.
\end{abstract}

\section{Introduction}

Let $(M, \nabla)$ be an $n$-dimensional differentiable manifold equipped with a torsionfree linear connection $\nabla$. Denote by $T^{*} M$ the cotangent bundle of $M$ and let $\pi$ be the natural projection $T^{*} M \rightarrow M$. The vertical distribution $V$ on $T^{*} M$ ( $V$ is the kernel of the submersion $T^{*} M \rightarrow M$ ), which is the integrable distribution. If $M$ is a paracompact manifold there exists a $C^{\infty}$-distribution $H$ on $T^{*} M$ which is supplementary to the vertical distribution $V$, such as the Whitney sum $T T^{*} M=$ $H T^{*} M \oplus V T^{*} M$ holds.

For the torsion-free linear connection $\nabla$ on $M$, the cotangent bundle of $M, T^{*} M$, can be endowed with a pseudo-Riemannian metric $\bar{g}_{\nabla}$ of neutral signature, called the Riemannian extension of $\nabla$, given by

$$
\begin{aligned}
\bar{g}_{\nabla}\left({ }^{H} X,{ }^{H} Y\right) & =0 \\
\bar{g}_{\nabla}\left({ }^{H} X,{ }^{V} \omega\right) & =\bar{g}_{\nabla}\left({ }^{V} \omega,{ }^{H} X\right)=\omega(X) \\
\bar{g}_{\nabla}\left({ }^{V} \omega,{ }^{V} \theta\right) & =0
\end{aligned}
$$

where ${ }^{H} X$ and ${ }^{H} Y$ denote the horizontal lifts of the vector fields $X$ and $Y$, and ${ }^{V} \omega$ and ${ }^{V} \theta$ denote the vertical lifts of the covectors (1-forms) $\omega$ and $\theta$. Thus, the Riemannian extension of $(M, \nabla)$ is a pseudo-Riemannian manifold $\left(T^{*} M, \bar{g}_{\nabla}\right)$. Riemannian extensions were first defined and studied by Patterson and Walker [16] and then investigated in Afifi [2]. Moreover, Riemannian extensions were also considered in Garcia-Rio et al. 7] in relation to Osserman manifolds (see also Derdzinski [5]). For further references relation to Riemannian extensions, see [1, 6. 10, 15, 21, 22, 23. Classical Riemannian extensions have been generalized in 
several ways, see, as an example [13. In [3, 4, the authors introduced another generalization which is called modified Riemannian extension. For a symmetric $(0,2)$-tensor field $c$ on $(M, \nabla)$, this metric is given by $\bar{g}_{\nabla, c}=\bar{g}_{\nabla}+\pi^{*} c$, that is,

$$
\begin{aligned}
\bar{g}_{\nabla, c}\left({ }^{H} X,{ }^{H} Y\right) & =c(X, Y) \\
\bar{g}_{\nabla, c}\left({ }^{H} X,{ }^{V} \omega\right) & =\bar{g}_{\nabla, c}\left({ }^{V} \omega,{ }^{H} X\right)=\omega(X) \\
\bar{g}_{\nabla, c}\left({ }^{V} \omega,{ }^{V} \theta\right) & =0 .
\end{aligned}
$$

In this paper, we consider a metric connection $\widetilde{\nabla}$ with nonvanishing torsion on the cotangent bundle $T^{*} M$ with modified Riemannian extension $\bar{g}_{\nabla, c}$. First, we give a necessary and sufficient condition for a vector field on $\left(T^{*} M, \bar{g}_{\nabla, c}\right)$ to be fibrepreserving projective vector field on $T^{*} M$ with respect to the metric connection $\widetilde{\nabla}$. This condition is represented by a set of relations involving certain tensor fields on $M$. Secondly, we investigate the conditions for the cotangent bundle $\left(T^{*} M, \bar{g}_{\nabla, c}\right)$ to be semi-symmetric, Ricci semi-symmetric, $\widetilde{Z}$ semi-symmetric and locally conharmonically flat with respect to the metric connection $\widetilde{\nabla}$. Finally, we show that the Schouten-Van Kampen connection associated to the Levi-Civita connection $\bar{\nabla}$ of the modified Riemannian extension $\bar{g}_{\nabla, c}$ is equal to the horizontal lift ${ }^{H} \nabla$ of the torsion-free linear connection $\nabla$ to $T^{*} M$ and present a result concerning the curvature tensor of the Schouten-Van Kampen connection.

The manifolds, tensor fields and geometric objects we consider in this paper are assumed to be differentiable of class $C^{\infty}$. Einstein's summation convention is used, the range of the indices $h, i, j, k, l, m, r$, being always $\{1, \ldots, n\}$.

\section{Preliminaries}

We refer to 24 for further details concerning the material of this section. Let $M$ be an $n$-dimensional differentiable manifold with a torsion-free linear connection $\nabla$ and denote by $\pi: T^{*} M \rightarrow M$ its cotangent bundle with fibres the cotangent spaces to $M$. Then $T^{*} M$ is a $2 n$-dimensional smooth manifold and some local charts induced naturally from local charts on $M$, may be used. Namely, a system of local coordinates $\left(U, x^{i}\right), i=1, \ldots, n$ on $M$ induces on $T^{*} M$ a system of local coordinates $\left(\pi^{-1}(U), x^{i}, x^{\bar{i}}=p_{i}\right), \bar{i}=n+i=n+1, \ldots, 2 n$, where $x^{\bar{i}}=p_{i}$ is the components of covectors $p$ in each cotangent space $T_{x}^{*} M, x \in U$ with respect to the natural coframe $\left\{d x^{i}\right\}$.

Let $X=X^{i} \frac{\partial}{\partial x^{i}}$ and $\omega=\omega_{i} d x^{i}$ be the local expressions in $U$ of a vector field $X$ and a covector field $\omega$ on $M$, respectively. Then the vertical lift $V \omega$ of $\omega$, the horizontal lift ${ }^{H} X$ of $X$ are given, with respect to the induced coordinates, by

$$
{ }_{\omega} \omega=\omega_{i} \partial_{\bar{i}}
$$

and

$$
{ }^{H} X=X^{i} \partial_{i}+p_{h} \Gamma_{i j}^{h} X^{j} \partial_{\bar{i}}
$$

where $\partial_{i}=\frac{\partial}{\partial x^{i}}, \partial_{\bar{i}}=\frac{\partial}{\partial x^{\bar{i}}}$ and $\Gamma_{i j}^{h}$ are the coefficients of $\nabla$ on $M$.

Next, we can introduce a frame field on each induced coordinate neighborhood $\pi^{-1}(U)$ of $T^{*} M$. It is called the adapted frame and consists of the following $2 n$ 
linearly independent vector fields $\left\{E_{\beta}\right\}=\left\{E_{j}, E_{\bar{j}}\right\}$ :

$$
\left\{\begin{array}{c}
E_{j}=\partial_{j}+p_{a} \Gamma_{h j}^{a} \partial_{\bar{h}} \\
E_{\bar{j}}=\partial_{\bar{j}} .
\end{array}\right.
$$

The indices $\alpha, \beta, \gamma, \ldots=1, \ldots, 2 n$ indicate the indices with respect to the adapted frame. The Lie brackets of the adapted frame of $T^{*} M$ satisfy the following identities:

$$
\left\{\begin{array}{c}
{\left[E_{i}, E_{j}\right]=p_{s} R_{i j l}^{s} E_{\bar{l}}} \\
{\left[E_{i}, E_{\bar{j}}\right]=-\Gamma_{i l}^{j} E_{\bar{l}}} \\
{\left[E_{\bar{i}}, E_{\bar{j}}\right]=0}
\end{array}\right.
$$

where $R_{i j l}{ }^{s}$ denote the coefficients of the curvature tensor $R$ of $\nabla$ on $M$.

With respect to the adapted frame $\left\{E_{\beta}\right\}$, the vector fields ${ }^{V} \omega$ and ${ }^{H} X$ on $T^{*} M$ has the components

$$
{ }^{V} \omega=\left(\begin{array}{l}
0 \\
\omega_{j}
\end{array}\right) \text { and }{ }^{H} X=\left(\begin{array}{l}
X^{j} \\
0
\end{array}\right)
$$

\section{THE METRIC CONNECTION WITH NONVANISHING TORSION ON THE}

COTANGENT BUNDLE WITH RESPECT TO MODIFIED RIEMANNIAN EXTENSION

Let us consider $T^{*} M$ equipped with the modified Riemannian extension $\bar{g}_{\nabla, c}$ for a given torsion-free connection $\nabla$ on $M$. In adapted frame $\left\{E_{\beta}\right\}$, the modified Riemannian extension $\left(\bar{g}_{\nabla, c}\right)_{\beta \gamma}$ and its inverse $\left(\bar{g}_{\nabla, c}\right)^{\beta \gamma}$ have in the following forms:

$$
\begin{aligned}
& \left(\bar{g}_{\nabla, c}\right)_{\beta \gamma}=\left(\begin{array}{cc}
c_{i j} & \delta_{i}^{j} \\
\delta_{j}^{i} & 0
\end{array}\right) . \\
& \left(\bar{g}_{\nabla, c}\right)^{\beta \gamma}=\left(\begin{array}{cc}
0 & \delta_{j}^{i} \\
\delta_{i}^{j} & -c_{i j}
\end{array}\right)
\end{aligned}
$$

where $c_{i j}$ are the components of the symmetric $(0,2)$-tensor field $c$ on $(M, \nabla)$.

For the Levi-Civita connection $\bar{\nabla}$ of the modified Riemannian extension $\bar{g}_{\nabla, c}$, we get:

Proposition 1. Let $\nabla$ be a torsion-free linear connection on $M$ and $T^{*} M$ be the cotangent bundle with the modified Riemann extension $\bar{g}_{\nabla, c}$ over $(M, \nabla)$. The LeviCivita connection $\bar{\nabla}$ of $\left(T^{*} M, \bar{g}_{\nabla, c}\right)$ is given by

$$
\left\{\begin{array}{c}
\bar{\nabla}_{E_{\bar{z}} E_{\bar{j}}}=0, \bar{\nabla}_{E_{\bar{i}}} E_{j}=0, \\
\bar{\nabla}_{E_{i}} E_{\bar{j}}=-\Gamma_{i h}^{j} E_{\bar{h}}, \\
\bar{\nabla}_{E_{i}} E_{j}=\Gamma_{i j}^{h} E_{h}+\left\{p_{s} R_{h j i}{ }^{s}+\frac{1}{2}\left(\nabla_{i} c_{j h}+\nabla_{j} c_{i h}-\nabla_{h} c_{i j}\right)\right\} E_{\bar{h}}
\end{array}\right.
$$

with respect to the adapted frame $\left\{E_{\beta}\right\}$, where $\Gamma_{i j}^{h}$ and $R_{h j i}{ }^{s}$ respectively denote components of $\nabla$ and its curvature tensor field $R$ on $M$ (see, [8]). 
If there is a Riemannian metric $g$ on $M$ such that $\nabla g=0$, then the connection $\nabla$ is a metric connection, otherwise it is non-metric. It is well known that a linear connection is symmetric and metric if and only if it is the Levi-Civita connection. The Levi-Civita connection $\bar{\nabla}$ of the modified Riemannian extension $\bar{g}_{\nabla, c}$ on $T^{*} M$ is the unique connection which satisfies $\bar{\nabla}_{\alpha}\left(\bar{g}_{\nabla, c}\right)_{\beta \gamma}=0$ and has a zero torsion. Now we are interested in a metric connection $\widetilde{\nabla}$ of the modified Riemannian extension $\bar{g}_{\nabla, c}$ whose torsion tensor $\widetilde{T}_{\gamma \beta}^{\varepsilon}$ is skew-symmetric in the indices $\gamma$ and $\beta$. Metric connection with nonvanishing torsion on Riemannian manifolds were introduced by Hayden 9 . We denote components of the metric connection $\widetilde{\nabla}$ by $\widetilde{\Gamma}_{\alpha \beta}^{\gamma}$. The metric connection $\widetilde{\nabla}$ satisfies

$$
\widetilde{\nabla}_{\alpha}\left(\bar{g}_{\nabla, c}\right)_{\beta \gamma}=0 \text { and } \widetilde{\Gamma}_{\alpha \beta}^{\gamma}-\widetilde{\Gamma}_{\beta \alpha}^{\gamma}=\widetilde{T}_{\alpha \beta}^{\gamma} .
$$

When the above equation is solved with respect to $\widetilde{\Gamma}_{\alpha \beta}^{\gamma}$, one finds the following solution 9

$$
\widetilde{\Gamma}_{\alpha \beta}^{\gamma}=\bar{\Gamma}_{\alpha \beta}^{\gamma}+\widetilde{U}_{\alpha \beta}^{\gamma},
$$

where $\bar{\Gamma}_{\alpha \beta}^{\gamma}$ is the components of the Levi-Civita connection $\bar{\nabla}$ of the modified Riemannian extension $\bar{g}_{\nabla, c}$,

$$
\widetilde{U}_{\alpha \beta \gamma}=\frac{1}{2}\left(\widetilde{T}_{\alpha \beta \gamma}+\widetilde{T}_{\gamma \alpha \beta}+\widetilde{T}_{\gamma \beta \alpha}\right)
$$

and

$$
\widetilde{U}_{\alpha \beta \gamma}=\widetilde{U}_{\alpha \beta}^{\epsilon}\left(\bar{g}_{\nabla, c}\right)_{\epsilon \gamma}, \widetilde{T}_{\alpha \beta \gamma}=\widetilde{T}_{\alpha \beta}^{\epsilon}\left(\bar{g}_{\nabla, c}\right)_{\epsilon \gamma} .
$$

If we choose the torsion tensor $\widetilde{T}$ as

$$
\left\{\begin{array}{l}
\widetilde{T}_{i j}^{\bar{r}}=-p_{s} R_{i j r}{ }^{s} \\
\text { otherwise }=0
\end{array}\right.
$$

with the help of (3.6), from (3.5), we find non-zero component of $\widetilde{U}_{\alpha \beta}^{\gamma}$ as follows:

$$
\widetilde{U}_{i j}^{\bar{h}}=p_{s} R_{j h i}^{s}
$$

with respect to the adapted frame. In view of (3.3) and (3.4), we have the following proposition.

Proposition 2. Let $\nabla$ be a torsion-free linear connection on $M$ and $T^{*} M$ be the cotangent bundle with the modified Riemann extension $\bar{g}_{\nabla, c}$ over $(M, \nabla)$. The metric connection $\widetilde{\nabla}$ on $T^{*} M$ with respect to the modified Riemannian extension $\bar{g}_{\nabla, c}$ satisfies

$$
\left\{\begin{array}{c}
\widetilde{\nabla}_{E_{\bar{\tau}}} E_{\bar{j}}=0, \widetilde{\nabla}_{E_{\bar{z}}} E_{j}=0, \\
\widetilde{\nabla}_{E_{i}} E_{\bar{j}}=-\Gamma_{i h}^{j} E_{\bar{h}}, \\
\widetilde{\nabla}_{E_{i}} E_{j}=\Gamma_{i j}^{h} E_{h}+\frac{1}{2}\left(\nabla_{i} c_{j h}+\nabla_{j} c_{i h}-\nabla_{h} c_{i j}\right) E_{\bar{h}}
\end{array}\right.
$$

with respect to the adapted frame $\left\{E_{\beta}\right\}$.

The horizontal lift ${ }^{H} \nabla$ of the torsion-free linear connection $\nabla$ on $M$ to $T^{*} M$ is characterized the following conditions:

$$
\left\{\begin{array}{c}
{ }^{H} \nabla_{V}{ }_{\omega}{ }^{V} \theta=0,{ }^{H} \nabla_{V \omega}{ }^{H} Y=0 \\
{ }^{H} \nabla^{H} X \\
{ }^{V} \theta={ }^{V}\left(\nabla_{X} \theta\right),{ }^{H} \nabla^{H} X
\end{array}\right.
$$


for all vector fields $X, Y$ and covector fields $\omega, \theta$ on $M$ ([24], p. 287). In the adapted frame, the followings satisfy (see, also [1])

$$
\left\{\begin{array}{c}
{ }^{H} \nabla_{E_{\bar{i}}} E_{\bar{j}}=0,{ }^{H} \nabla_{E_{\bar{\tau}}} E_{j}=0, \\
{ }^{H} \nabla_{E_{i}} E_{\bar{j}}=-\Gamma_{i h}^{j} E_{\bar{h}},{ }^{H} \nabla_{E_{i}} E_{j}=\Gamma_{i j}^{h} E_{h} .
\end{array}\right.
$$

From these formulas, we can readily deduce:

Proposition 3. Let $\nabla$ be a torsion-free linear connection on $M$ and $T^{*} M$ be the cotangent bundle with the modified Riemann extension $\bar{g}_{\nabla, c}$ over $(M, \nabla)$. The metric connection $\widetilde{\nabla}$ on $T^{*} M$ of the modified Riemannian extension $\bar{g}_{\nabla, c}$ coincides with the horizontal lift ${ }^{H} \nabla$ of the torsion-free linear connection $\nabla$ on $M$ if and only if the components $c_{i j}$ of $c$ satisfy the condition

$$
\nabla_{i} c_{j h}+\nabla_{j} c_{i h}-\nabla_{h} c_{i j}=0
$$

3.1. Projective vector fields on the cotangent bundle with respect to the metric connection $\widetilde{\nabla}$. Given a linear connection $\nabla$ on a manifold $M$, a vector field $V$ is said to be a projective vector field if there exists a 1 -form $\theta$ such that

$$
\left(L_{V} \nabla\right)(X, Y)=\theta(X) Y+\theta(Y) X
$$

for any pair of vector fields $X$ and $Y$ on $M$. In particular, if $\theta=0, V$ is an affine Killing vector field.

Let $\widetilde{V}$ be a vector field on $T^{*} M$ and $\left(v^{h}, v^{\bar{h}}\right)$ its the components with respect to the adapted frame $\left\{E_{\beta}\right\}$. The components $v^{h}$ and $v^{\bar{h}}$ are said to be the horizontal components and vertical components of $\widetilde{V}$, respectively. As is known, a vector field is called a fibre-preserving vector field if and only if its horizontal components depend only on the variables $\left(x^{h}\right)$. Hence, one can easily say that every fibrepreserving vector field $\widetilde{V}$ on $T^{*} M$ induces a vector field $V$ with components $\left(v^{h}\right)$ on the base manifold $M$.

By straightforward calculations, we have the following.

Lemma 1. Let $\widetilde{V}$ be a fibre-preserving vector field on $T^{*} M$ with components $\left(v^{h}, v^{\bar{h}}\right)$. The Lie derivatives of the adapted frame satisfy

$$
\begin{aligned}
i) L_{\widetilde{V}} E_{i} & =-\left(E_{i} v^{k}\right) E_{k}-\left(v^{a} p_{s} R_{i a k} s+E_{i} v^{\bar{k}}-v^{\bar{a}} \Gamma_{i k}^{a}\right) E_{\bar{k}}, \\
i i) L_{\widetilde{V}} E_{\bar{i}} & =-\left(v^{a} \Gamma_{a k}^{i}+E_{\bar{i}} v^{\bar{k}}\right) E_{\bar{k}},
\end{aligned}
$$

where $L_{\widetilde{V}}$ denotes the Lie derivation with respect to $\widetilde{V}$.

The general forms of fibre-preserving projective vector fields on $T^{*} M$ with respect to the metric connection $\widetilde{\nabla}$ are given by:

Theorem 1. Let $\nabla$ be a torsion-free linear connection on $M$ and $T^{*} M$ be the cotangent bundle with the modified Riemannian extension $\bar{g}_{\nabla, c}$ over $(M, \nabla)$. Then a vector field $\widetilde{V}$ is a fibre-preserving projective vector field with associated 1 -form $\widetilde{\theta}$ on $T^{*} M$ with respect to the metric connection $\widetilde{\nabla}$ if and only if the vector field $\widetilde{V}$ is defined by

$$
\widetilde{X}={ }^{H} V+{ }^{V} B+\gamma A
$$

where the vector field $V=\left(v^{h}\right)$, the covector field $B=\left(B_{h}\right)$, the $(1,1)$-tensor field $A=\left(A_{i}^{h}\right)$ and the associated $1-$ form $\widetilde{\theta}$ satisfy 
(i) $\widetilde{\theta}=\theta_{i} d x^{i}$,

(ii) $\nabla_{j} A_{k}^{i}=\theta_{j} \delta_{i}^{k}-v^{a} R_{j a k}^{i}$,

(iii) $L_{V} \Gamma_{i j}^{h}=\theta_{i} \delta_{j}^{h}+\theta_{j} \delta_{i}^{h}$

(iv) $\nabla_{i} \nabla_{j} B_{l}+R_{l j i}{ }^{a} B_{a}+\frac{1}{2} v^{a} \nabla_{a} M_{i j l}+\frac{1}{2}\left(\nabla_{j} v^{a}\right) M_{i a l}$

$+\frac{1}{2}\left(\nabla_{i} v^{a}\right) M_{a j l}-A_{l}^{a} M_{i j a}=0 \quad\left(M_{i j l}:=\nabla_{i} c_{j l}+\nabla_{j} c_{i l}-\nabla_{l} c_{i j}\right)$

(v) $\nabla_{i} \nabla_{j} A_{l}^{s}+R_{l j i}{ }^{a} A_{a}^{s}-R_{a j i}{ }^{s} A_{l}^{a}+v^{a} \nabla_{i} R_{j a l}{ }^{s}+\left(\nabla_{i} v^{a}\right) R_{j a l}{ }^{s}=0$.

Proof. A fibre-preserving vector field $\widetilde{V}=v^{h} E_{h}+v^{\bar{h}} E_{\bar{h}}$ on $T^{*} M$ is a fibre-preserving projective vector field if and only if there exist a 1 -form $\widetilde{\theta}$ with components $\left(\widetilde{\theta}_{i}, \widetilde{\theta}_{\bar{i}}\right)$ on $T^{*} M$ such that

$$
\begin{aligned}
\left(L_{\widetilde{V}} \widetilde{\nabla}\right)(\widetilde{Y}, \widetilde{Z}) & =L_{\widetilde{V}}\left(\widetilde{\nabla}_{\widetilde{Y}} \widetilde{Z}\right)-\widetilde{\nabla}_{\widetilde{Y}}\left(L_{\widetilde{V}} \widetilde{Z}\right)-\widetilde{\nabla}_{\left(L_{\tilde{V}} \widetilde{Y}\right)} \widetilde{Z} \\
& =\widetilde{\theta}(\widetilde{Y}) \widetilde{Z}+\widetilde{\theta}(\widetilde{Z}) \widetilde{Y}
\end{aligned}
$$

for any vector fields $\tilde{Y}$ and $\widetilde{Z}$ on $T^{*} M$.

Putting $\widetilde{Y}=E_{\bar{i}}, \widetilde{Z}=E_{\bar{j}}$ in (3.9), we get

$$
E_{\bar{i}}\left(E_{\bar{j}} v^{\bar{k}}\right) E_{\bar{k}}=\theta_{\bar{i}} E_{\bar{j}}+\theta_{\bar{j}} E_{\bar{i}} .
$$

Putting $Y=E_{\bar{i}}, Z=E_{j}$ in (3.9), we find

$$
\theta_{\bar{i}}=0
$$

and

$$
v^{a} R_{j a k}^{i}+E_{\bar{i}}\left(E_{j} v^{\bar{k}}\right)-\left(E_{\bar{i}} v^{\bar{a}}\right) \Gamma_{j k}^{a}=\theta_{j} \delta_{i}^{k} .
$$

In view of (3.11), (3.10) reduces to

from which it follows that

$$
E_{\bar{i}}\left(E_{\bar{j}} v^{\bar{k}}\right) E_{\bar{k}}=0
$$

$$
v^{\bar{k}}=p_{s} A_{k}^{s}+B_{k}
$$

where $A_{k}^{s}$ and $B_{k}$ are certain functions which depend only on the variables $\left(x^{h}\right)$. The coordinate transformation rule implies that $A$ is a $(1,1)$-tensor field with components $\left(A_{k}^{s}\right)$ and $B$ is a covector field with components $\left(B_{k}\right)$. Hence, the fibre-preserving projective vector field $\widetilde{V}$ on $T^{*} M$ can be written in the form:

$$
\begin{aligned}
\widetilde{V} & =v^{k} E_{k}+v^{\bar{k}} E_{\bar{k}}=v^{k} E_{k}+\left\{p_{s} A_{k}^{s}+B_{k}\right\} E_{\bar{k}} \\
& ={ }^{H} V+{ }^{V} B+\gamma A
\end{aligned}
$$

where $\gamma$ is an operator applied to the $(1,1)$-tensor field $A$ and expressed locally $\gamma A=\left(p_{s} A_{k}^{s}\right) E_{\bar{k}}$ (for details related to the operator $\gamma$, see [24, p.12-13).

Substitution (3.13) into (3.12) gives

$$
v^{a} R_{j a k}^{i}+\nabla_{j} A_{k}^{i}=\theta_{j} \delta_{i}^{k} .
$$

Contracting $i$ and $k$ in (3.14), we have

$$
\theta_{j}=\frac{1}{n} \nabla_{j} A_{k}^{k}
$$

Finally, putting $Y=E_{i}, Z=E_{j}$ in (3.9), we obtain

$$
L_{V} \Gamma_{i j}^{h}=\theta_{i} \delta_{j}^{h}+\theta_{j} \delta_{i}^{h}
$$




$$
\begin{aligned}
& \nabla_{i} \nabla_{j} B_{l}-R_{i j l}^{a} B_{a}+\frac{1}{2} v^{a} \nabla_{a} M_{i j l} \\
& +\frac{1}{2}\left(\nabla_{j} v^{a}\right) M_{i a l}+\frac{1}{2}\left(\nabla_{i} v^{a}\right) M_{a j l}-A_{l}^{a} M_{i j a} \\
= & 0
\end{aligned}
$$

and

where $M_{i j l}=\nabla_{i} c_{j l}+\nabla_{j} c_{i l}-\nabla_{l} c_{i j}$.

$$
\nabla_{i} \nabla_{j} A_{l}^{s}+R_{l j i}^{a} A_{a}^{s}-R_{a j i}^{s} A_{l}^{a}+v^{a} \nabla_{i} R_{j a l}^{s}+\left(\nabla_{i} v^{a}\right) R_{j a l}^{s}=0,
$$

Conversely, if $B_{h}, v^{h}, \theta_{h}$ and $A_{i}^{h}$ are given so that they satisfy $(i)-(v)$, reversing the above steps, we see that $\widetilde{V}={ }^{H} V+{ }^{V} B+\gamma A$ is a fibre-preserving projective vector field on $T^{*} M$ with respect to the metric connection $\widetilde{\nabla}$. This completes the proof.

The below result follows immediately from Theorem 1 and its Proof.

Corollary 1. Let $\nabla$ be a torsion-free linear connection on $M$ and $T^{*} M$ be the cotangent bundle with the modified Riemannian extension $\bar{g}_{\nabla, c}$ over $(M, \nabla)$. Every fibre-preserving projective vector field $\widetilde{V}$ with respect to the metric connection $\widetilde{\nabla}$ is of the form (3.8) and it naturally induces a projective vector field $V$ on $M$.

3.2. Semi-Symmetry properties of the cotangent bundle with respect to the metric connection $\widetilde{\nabla}$. Given a manifold $M(\operatorname{dim}(M) \geq 3)$ endowed with a linear connection $\nabla$ whose curvature tensor is marked as $R$, for any tensor field of $S$ of type $(0, k), k \geq 1$, the tensor field $R(X, Y) . S$ is expressed in the form:

$$
\begin{aligned}
(R(X, Y) . S)\left(X_{1}, X_{2}, \ldots, X_{k}\right)= & -S\left(R(X, Y) X_{1}, X_{2}, \ldots, X_{k}\right) \\
& -\ldots-S\left(X_{1}, X_{2}, \ldots, X_{k-1}, R(X, Y) X_{k}\right)
\end{aligned}
$$

for any vector fields $X_{1}, X_{2}, \ldots, X_{k}, X, Y$ on $M$, where $R(X, Y)$ acts as a derivation on $S$. If $R(X, Y) . S=0$, then the manifold $M$ is said to be $S$ semi-symmetric with respect to the linear connection $\nabla$. A (pseudo-) Riemannian manifold $(M, g)$ such that its curvature tensor $R$ satisfies the condition

$$
R(X, Y) \cdot R=0
$$

is called a semi-symmetric space. Also, note that locally symmetric spaces are semisymmetric, but in general the converse is not true. The semi-symmetric space was first studied by Cartan. Nevertheless, Sinjukov first used the name "semi-symmetric spaces" for manifolds satisfying the above curvature condition [18. Later, Szabo gave the full local and global classification of semi-symmetric spaces [19, 20. A (pseudo-)Riemannian manifold $(M, g)$ is called Ricci semi-symmetric if the following condition is satisfied:

$$
R(X, Y) \cdot R i c=0,
$$

where Ric is the Ricci tensor of $(M, g)$. It is obvious that any semi-symmetric manifold is Ricci semi-symmetric.

The curvature tensor $\widetilde{R}$ of the metric connection $\widetilde{\nabla}$ on $T^{*} M$ is obtained from the formula

$$
\widetilde{R}\left(E_{\alpha}, E_{\beta}\right) E_{\gamma}=\widetilde{\nabla}_{E_{\alpha}} \widetilde{\nabla}_{E_{\beta}} E_{\gamma}-\widetilde{\nabla}_{E_{\beta}} \widetilde{\nabla}_{E_{\alpha}} E_{\gamma}-\widetilde{\nabla}_{\left[E_{\alpha}, E_{\beta}\right]} E_{\gamma}
$$

with respect to the adapted frame. For the curvature tensor $\widetilde{R}$ of the metric connection $\widetilde{\nabla}$, with the help of (2.1) and (3.7), we have: 
Proposition 4. Let $\nabla$ be a torsion-free linear connection on $M$ and $T^{*} M$ be the cotangent bundle with the modified Riemann extension $\bar{g}_{\nabla, c}$ over $(M, \nabla)$. The curvature tensor $\widetilde{R}$ of the metric connection $\widetilde{\nabla}$ on $T^{*} M$ satisfies the following conditions:

$$
\begin{aligned}
\widetilde{R}\left(E_{i}, E_{j}\right) E_{k}= & R_{i j k}{ }^{h} E_{h} \\
& +\frac{1}{2}\left\{\nabla_{i}\left(\nabla_{k} c_{j h}-\nabla_{h} c_{j k}\right)-\nabla_{j}\left(\nabla_{k} c_{i h}-\nabla_{h} c_{i k}\right)\right. \\
& \left.-R_{i j k}{ }^{m} c_{m h}-R_{i j h}{ }^{m} c_{k m}\right\} E_{\bar{h}} \\
\widetilde{R}\left(E_{i}, E_{j}\right) E_{\bar{k}}= & R_{j i h}{ }^{k} E_{\bar{h}}, \\
\widetilde{R}\left(E_{i}, E_{\bar{j}}\right) E_{k}= & 0, \widetilde{R}\left(E_{i}, E_{\bar{j}}\right) E_{\bar{k}}=0, \widetilde{R}\left(E_{\bar{i}}, E_{j}\right) E_{k}=0, \\
\widetilde{R}\left(E_{\bar{i}}, E_{j}\right) E_{\bar{k}}= & 0, \widetilde{R}\left(E_{\bar{i}}, E_{\bar{j}}\right) E_{k}=0, \widetilde{R}\left(E_{\bar{i}}, E_{\bar{j}}\right) E_{\bar{k}}=0
\end{aligned}
$$

with respect to the adapted frame $\left\{E_{\beta}\right\}$.

Let $\widetilde{X}$ and $\widetilde{Y}$ be vector fields of $T^{*} M$. The curvature operator $\widetilde{R}(\widetilde{X}, \widetilde{Y})$ is a differential operator on $T^{*} M$. Similarly, for vector fields $X$ and $Y$ of $M, R(X, Y)$ is a differential operator on $M$. Now, we operate the curvature operator $\widetilde{R}(\widetilde{X}, \widetilde{Y})$ to the curvature tensor $\widetilde{R}$. That is, for all $\widetilde{Z}, \widetilde{W}$ and $\widetilde{U}$, we consider the condition $(\widetilde{R}(\widetilde{X}, \widetilde{Y}) \widetilde{R})(\widetilde{Z}, \widetilde{W}) \widetilde{U}=0$. In the case, we shall call the cotangent bundle $T^{*} M$ as semi-symmetric with respect to the metric connection $\widetilde{\nabla}$.

In the adapted frame $\left\{E_{\beta}\right\}$, the tensor $(\widetilde{R}(\widetilde{X}, \widetilde{Y}) \widetilde{R})(\widetilde{Z}, \widetilde{W}) \widetilde{U}$ is locally expressed as follows:

$$
\begin{aligned}
& ((\widetilde{R}(\widetilde{X}, \widetilde{Y}) \widetilde{R})(\widetilde{Z}, \widetilde{W}) \widetilde{U})_{\alpha \beta \gamma \theta \sigma}{ }^{\varepsilon} \\
= & \widetilde{R}_{\alpha \beta \tau} \widetilde{R}_{\gamma \theta \sigma}^{\tau}-\widetilde{R}_{\alpha \beta \gamma}^{\tau} \widetilde{R}_{\tau \theta \sigma}^{\varepsilon}-\widetilde{R}_{\alpha \beta \theta}^{\tau} \widetilde{R}_{\gamma \tau \sigma}^{\varepsilon}-\widetilde{R}_{\alpha \beta \sigma}{ }^{\tau} \widetilde{R}_{\gamma \theta \tau}{ }^{\varepsilon} .
\end{aligned}
$$

Similarly, in local coordinates,

$$
\begin{aligned}
& ((R(X, Y) R)(Z, W) U)_{i j k l m}{ }^{n} \\
= & R_{i j p}{ }^{n} R_{k l m}{ }^{p}-R_{i j k}{ }^{p} R_{p l m}{ }^{n}-R_{i j l}^{p} R_{k p m}{ }^{n}-R_{i j m}{ }^{p} R_{k l p}{ }^{n} .
\end{aligned}
$$

Theorem 2. Let $\nabla$ be a torsion-free linear connection on $M$ and $T^{*} M$ be the cotangent bundle with the modified Riemannian extension $\bar{g}_{\nabla, c}$ over $(M, \nabla)$. Under the assumption that $\nabla_{i}\left(\nabla_{k} c_{j h}-\nabla_{h} c_{j k}\right)-\nabla_{j}\left(\nabla_{k} c_{i h}-\nabla_{h} c_{i k}\right)-R_{i j k}{ }^{m} c_{m h}-R_{i j h}{ }^{m} c_{k m}=$ 0 , where $R$ is the curvature tensor of $\nabla$, the cotangent bundle $T^{*} M$ is semisymmetric with respect to the metric connection $\widetilde{\nabla}$ if and only if the base manifold $M$ is semi-symmetric with respect to $\nabla$.

Proof. We consider the conditions $(\widetilde{R}(\widetilde{X}, \widetilde{Y}) \widetilde{R})(\widetilde{Z}, \widetilde{W}) \widetilde{U}=0$ for all vector fields $\widetilde{X}, \widetilde{Y}, \widetilde{Z}, \widetilde{W}$ and $\widetilde{U}$ on $T^{*} M$.

For all cases $\alpha=(i, \bar{i}), \beta=(j, \bar{j}), \gamma=(k, \bar{k}), \theta=(l, \bar{l}), \sigma=(m, \bar{m})$ and $\varepsilon=(h, \bar{h})$ in (3.15), the non-zero components of the tensor $((\widetilde{R}(\widetilde{X}, \widetilde{Y}) \widetilde{R})(\widetilde{Z}, \widetilde{W}) \widetilde{U})_{\alpha \beta \gamma \theta \sigma}{ }^{\varepsilon}$ are 
as follows:

$$
\begin{aligned}
& ((\widetilde{R}(\widetilde{X}, \widetilde{Y}) \widetilde{R})(\widetilde{Z}, \widetilde{W}) \widetilde{U})_{i j k l m} h \\
& =\widetilde{R}_{i j p}{ }^{h} \widetilde{R}_{k l m}{ }^{p}+\widetilde{R}_{i j \bar{p}}{ }^{h} \widetilde{R}_{k l m}{ }^{\bar{p}}-\widetilde{R}_{i j k}{ }^{p} \widetilde{R}_{p l m}{ }^{h}-\widetilde{R}_{i j k}{ }^{\bar{p}} \widetilde{R}_{\bar{p} l m}{ }^{h} \\
& -\widetilde{R}_{i j l}{ }^{p} \widetilde{R}_{k p m}{ }^{h}-\widetilde{R}_{i j l}{ }^{\bar{p}} \widetilde{R}_{k \bar{p} m}{ }^{h}-\widetilde{R}_{i j m}{ }^{p} \widetilde{R}_{k l p}{ }^{h}-\widetilde{R}_{i j m}{ }^{\bar{p}} \widetilde{R}_{k l \bar{p}}{ }^{h} \\
& =R_{i j p}{ }^{h} R_{k l m}{ }^{p}-R_{i j k}{ }^{p} R_{p l m}{ }^{h}-R_{i j l}{ }^{p} R_{k p m}{ }^{h}-R_{i j m}{ }^{p} R_{k l p}{ }^{h} \\
& =((R(X, Y) R)(Z, W) U)_{i j k l m}{ }^{h} \text {. } \\
& \text { ii) } \quad((\widetilde{R}(\widetilde{X}, \widetilde{Y}) \widetilde{R})(\widetilde{Z}, \widetilde{W}) \widetilde{U})_{i j k l \bar{m}} \bar{h} \\
& =\widetilde{R}_{i j p}{ }^{\bar{h}} \widetilde{R}_{k l \bar{m}}^{p}+\widetilde{R}_{i j \bar{p}}{ }^{\bar{h}} \widetilde{R}_{k l \bar{m}}^{\bar{p}}-\widetilde{R}_{i j k}^{p} \widetilde{R}_{p l \bar{m}}{ }^{\bar{h}}-\widetilde{R}_{i j k}{ }^{\bar{p}} \widetilde{R}_{\bar{p} l \bar{m}}{ }^{\bar{h}} \\
& -\widetilde{R}_{i j l}^{p} \widetilde{R}_{k p \bar{m}}{ }^{\bar{h}}-\widetilde{R}_{i j l}{ }^{\bar{p}} \widetilde{R}_{k \overline{p m}} \bar{h}-\widetilde{R}_{i j \bar{m}}^{p} \widetilde{R}_{k l p}{ }^{\bar{h}}-\widetilde{R}_{i j \bar{m}} \bar{p} \widetilde{R}_{k l p}{ }^{\bar{h}} \\
& =-R_{i j p}{ }^{m} R_{k l h}{ }^{p}+R_{i j h}{ }^{p} R_{k l p}{ }^{m}+R_{i j k}{ }^{p} R_{p l h}{ }^{m}+R_{i j l}{ }^{p} R_{k p h}{ }^{m} \\
& =-((R(X, Y) R)(Z, W) U)_{i j k l h}{ }^{m} \text {. } \\
& \text { iii) }((\widetilde{R}(\widetilde{X}, \widetilde{Y}) \widetilde{R})(\widetilde{Z}, \widetilde{W}) \widetilde{U})_{i j k l m} \bar{h} \\
& =\widetilde{R}_{i j p}{ }^{h} \widetilde{R}_{k l m}^{p}+\widetilde{R}_{i j \bar{p}}{ }^{\bar{h}} \widetilde{R}_{k l m}{ }^{\bar{p}}-\widetilde{R}_{i j k}{ }^{p} \widetilde{R}_{p l m}{ }^{\bar{h}}-\widetilde{R}_{i j k}{ }^{\bar{p}} \widetilde{R}_{\bar{p} l m}{ }^{h} \\
& -\widetilde{R}_{i j l}^{p}{ }^{p} \widetilde{R}_{k p m}{ }^{\bar{h}}-\widetilde{R}_{i j l}{ }^{\bar{p}} \widetilde{R}_{k \bar{p} m}{ }^{\bar{h}}-\widetilde{R}_{i j m}^{p} \widetilde{R}_{k l p}{ }^{\bar{h}}-\widetilde{R}_{i j m}{ }^{\bar{p}} \widetilde{R}_{k l \bar{p}} \bar{h}
\end{aligned}
$$

If we assume that

$$
\begin{aligned}
\widetilde{R}_{i j k}^{\bar{h}}= & \nabla_{i}\left(\nabla_{k} c_{j h}-\nabla_{h} c_{j k}\right)-\nabla_{j}\left(\nabla_{k} c_{i h}-\nabla_{h} c_{i k}\right) \\
& -R_{i j k}{ }^{m} c_{m h}-R_{i j h}{ }^{m} c_{k m}=0,
\end{aligned}
$$

then it follows from (3.16) that $(\widetilde{R}(\widetilde{X}, \widetilde{Y}) \widetilde{R})(\widetilde{Z}, \widetilde{W}) \widetilde{U}=0$ if and only if $(R(X, Y) R)(Z, W) U=$ 0 . This completes the proof.

Denote by $\widetilde{R}_{\alpha \beta}=\widetilde{R}_{\sigma \alpha \beta}{ }^{\sigma}$ the contracted curvature tensor (Ricci tensor) of the metric connection $\widetilde{\nabla}$. The only non-zero component of $\widetilde{R}_{\alpha \beta}$ is as follows: $\widetilde{R}_{i j}=R_{i j}$, where $R_{i j}$ denote the components of the Ricci tensor of $\nabla$ on $M$. Now we prove the following theorem.

Theorem 3. Let $\nabla$ be a torsion-free linear connection on $M$ and $T^{*} M$ be the cotangent bundle with the modified Riemannian extension $\bar{g}_{\nabla, c}$ over $(M, \nabla)$. The cotangent bundle $T^{*} M$ is Ricci semi-symmetric with respect to the metric connection $\widetilde{\nabla}$ if and only if the base manifold $M$ is Ricci semi-symmetric with respect to $\nabla$.

Proof. The tensor $(\widetilde{R}(\widetilde{X}, \widetilde{Y}) \widetilde{R i c})(\widetilde{Z}, \widetilde{W})$ has the components

$$
((\widetilde{R}(\widetilde{X}, \widetilde{Y}) \widetilde{R i c})(\widetilde{Z}, \widetilde{W}))_{\alpha \beta \gamma \theta}=\widetilde{R}_{\alpha \beta \gamma}^{\varepsilon} \widetilde{R}_{\varepsilon \theta}+\widetilde{R}_{\alpha \beta \theta} \widetilde{R}_{\gamma \varepsilon}
$$

with respect to the adapted frame $\left\{E_{\beta}\right\}$.

Choosing $\alpha=i, \beta=j, \gamma=k, \theta=l$ in (3.16), we find

$$
\begin{aligned}
((\widetilde{R}(\widetilde{X}, \widetilde{Y}) \widetilde{R i c})(\widetilde{Z}, \widetilde{W}))_{i j k l} & =\widetilde{R}_{i j k}^{p} \widetilde{R}_{p l}+\widetilde{R}_{i j l}^{p} \widetilde{R}_{k p} \\
& =R_{i j k}^{p} R_{p l}+R_{i j l}^{p} R_{k p} \\
& =((R(X, Y) R i c)(Z, W))_{i j k l}
\end{aligned}
$$

all the others being zero. This finishes the proof. 
For the scalar curvature $\widetilde{r}$ of the metric connection $\widetilde{\nabla}$ with respect to $\bar{g}_{\nabla, c}$, with the help of (3.2) we find

$$
\widetilde{r}=\widetilde{R}_{\alpha \beta}\left(\bar{g}_{\nabla, c}\right)^{\alpha \beta}=0
$$

Thus we have the following theorem.

Theorem 4. Let $\nabla$ be a torsion-free linear connection on $M$ and $T^{*} M$ be the cotangent bundle with the modified Riemannian extension $\widetilde{g}_{\nabla, c}$ over $(M, \nabla)$. The scalar curvature of the cotangent bundle $T^{*} M$ with the metric connection $\widetilde{\nabla}$ with respect to $\widetilde{g}_{\nabla, c}$ vanishes.

Next, we shall apply the differential operator $\widetilde{R}(\widetilde{X}, \widetilde{Y})$ to the torsion tensor $\widetilde{T}$ of the metric connection $\widetilde{\nabla}$.

Theorem 5. Let $\nabla$ be a torsion-free linear connection on $M$ and $T^{*} M$ be the cotangent bundle with the modified Riemannian extension $\bar{g}_{\nabla, c}$ over $(M, \nabla)$. Then $\widetilde{R}(\widetilde{X}, \widetilde{Y}) . \widetilde{T}=0$ for all vector fields $\widetilde{X}$ and $\widetilde{Y}$ on $T^{*} M$, where $\widetilde{T}$ is the torsion tensor of the metric connection $\widetilde{\nabla}$ if and only if the base manifold $M$ is semi-symmetric with respect to $\nabla$.

Proof. The differential operator $\widetilde{R}(\widetilde{X}, \widetilde{Y})$ applied the torsion tensor $\widetilde{T}$ of the metric connection $\widetilde{\nabla}$ is in the form:

$$
\begin{aligned}
& ((\widetilde{R}(\widetilde{X}, \widetilde{Y}) \widetilde{T})(\widetilde{Z}, \widetilde{W}))_{\alpha \beta \gamma \theta}{ }^{\varepsilon} \\
= & \widetilde{R}_{\alpha \beta \tau} \widetilde{T}_{\gamma \theta}{ }^{\tau}-\widetilde{R}_{\alpha \beta \gamma} \widetilde{T}_{\tau \theta}{ }^{\varepsilon}-\widetilde{R}_{\alpha \beta \theta}{ }^{\tau} \widetilde{T}_{\gamma \tau} \varepsilon
\end{aligned}
$$

with respect to the adapted frame $\left\{E_{\beta}\right\}$. It follows immediately that

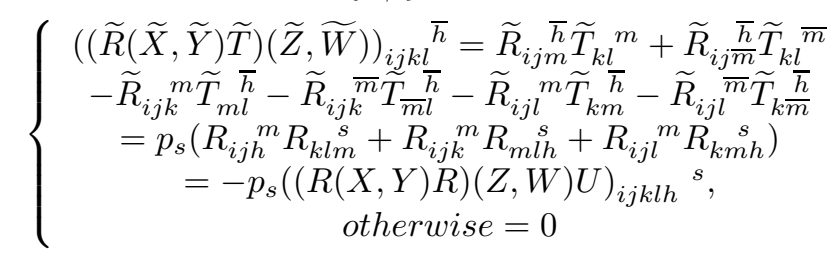

which finishes the proof.

On an $n$-dimensional Riemannian manifold $(M, g)$, it was defined a generalized $(0,2)$-symmetric $Z$ tensor given by 14

$$
Z(X, Y)=\operatorname{Ric}(X, Y)+\phi g(X, Y)
$$

for all vector fields $X$ and $Y$ on $M$, where where $\phi$ is an arbitrary scalar function. Analogous to this definition, it may be locally define generalized $\widetilde{Z}$ tensor on $\left(T^{*} M, \bar{g}_{\nabla, c}\right)$ with respect to the metric connection $\widetilde{\nabla}$ as follows:

$$
\widetilde{Z}_{\alpha \beta}=\widetilde{R}_{\alpha \beta}+\widetilde{\phi}\left(\bar{g}_{\nabla, c}\right)_{\alpha \beta}
$$

Putting the values of $\widetilde{R}_{\alpha \beta}$ and $\bar{g}_{\nabla, c}$ in the above equation, we have the non-zero components

$$
\begin{aligned}
\widetilde{Z}_{i j} & =R_{i j}+\widetilde{\phi} c_{i j}, \\
\widetilde{Z}_{\overline{i j}} & =\widetilde{\phi} \delta_{j}^{i}, \\
\widetilde{Z}_{i j} & =\widetilde{\phi} \delta_{j}^{i} .
\end{aligned}
$$

We can state the following theorem. 
Theorem 6. Let $\nabla$ be a torsion-free linear connection on $M$ and $T^{*} M$ be the cotangent bundle with the modified Riemannian extension $\bar{g}_{\nabla, c}$ over $(M, \nabla)$. The cotangent bundle $T^{*} M$ is $\widetilde{Z}$ semi-symmetric with respect to the metric connection $\widetilde{\nabla}$ if and only if the base manifold $M$ is Ricci semi-symmetric with respect to $\nabla$.

Proof. The tensor $(\widetilde{R}(\widetilde{X}, \widetilde{Y}) . \widetilde{Z})(\widetilde{Z}, \widetilde{W})$ has the components

$$
((\widetilde{R}(\widetilde{X}, \widetilde{Y}) \cdot \widetilde{Z})(\widetilde{Z}, \widetilde{W}))_{\alpha \beta \gamma \theta}=\widetilde{R}_{\alpha \beta \gamma}^{\varepsilon} \widetilde{Z}_{\varepsilon \theta}+\widetilde{R}_{\alpha \beta \theta}^{\varepsilon} \widetilde{Z}_{\gamma \varepsilon}
$$

with respect to the adapted frame $\left\{E_{\beta}\right\}$.

By choosing $\alpha=(i, \bar{i}), \beta=(j, \bar{j}), \gamma=(k, \bar{k})$ and $\theta=(l, \bar{l})$ in (3.19), in view of (3.18) we find the only non-zero component

$$
\begin{aligned}
& ((\widetilde{R}(\widetilde{X}, \widetilde{Y}) \cdot \widetilde{Z})(\widetilde{Z}, \widetilde{W}))_{i j k l} \\
= & \widetilde{R}_{i j k}{ }^{h} \widetilde{Z}_{h l}+\widetilde{R}_{i j k}{ }^{h} \widetilde{Z}_{\overline{h l}}+\widetilde{R}_{i j l}{ }^{h} \widetilde{Z}_{k h}+\widetilde{R}_{i j l}{ }^{h} \widetilde{Z}_{k \bar{h}} \\
= & R_{i j k}{ }^{h}\left(R_{h l}+\widetilde{\phi} c_{h l}\right)+\frac{1}{2}\left\{\nabla_{i}\left(\nabla_{k} c_{j h}-\nabla_{h} c_{j k}\right)\right. \\
& \left.-\nabla_{j}\left(\nabla_{k} c_{i h}-\nabla_{h} c_{i k}\right)-R_{i j k}{ }^{m} c_{m h}-R_{i j h}{ }^{m} c_{k m}\right\} \widetilde{\phi} \delta_{l}^{h} \\
& +R_{i j l}{ }^{h}\left(R_{k h}+\widetilde{\phi} c_{k h}\right)+\frac{1}{2}\left\{\nabla_{i}\left(\nabla_{l} c_{j h}-\nabla_{h} c_{j l}\right)\right. \\
& \left.-\nabla_{j}\left(\nabla_{l} c_{i h}-\nabla_{h} c_{i l}\right)-R_{i j l}{ }^{m} c_{m h}-R_{i j h}{ }^{m} c_{l m}\right\} \widetilde{\phi} \delta_{k}^{h} \\
= & R_{i j k}{ }^{h} R_{h l}+R_{i j l}{ }^{h} R_{k h} \\
= & (R(X, Y) R i c)_{i j k l},
\end{aligned}
$$

from which the proof follows.

3.3. Conharmonic Curvature tensor on the cotangent bundle with respect to the metric connection $\widetilde{\nabla}$. We recall that the conharmonic curvature tensor $V$ on an $n$-dimensional Riemannian manifold $(M, g)$ is defined as a $(4,0)$-tensor by the formula

$$
V_{i j k l}=R_{i j k l}-\frac{1}{n-2}\left[R_{j k} g_{i l}-R_{i k} g_{j l}-R_{j l} g_{i k}+R_{i l} g_{j k}\right],
$$

where $R_{i j k l}$ and $R_{i j}$ are respectively the components of the Riemannian curvature tensor and the Ricci tensor. The conharmonic curvature tensor was first introduced by Ishii (see, [12]). A Riemanian manifold whose conharmonic curvature tensor vanishes is called conharmonically flat.

Analogous to the conharmonic curvature tensor with respect to a Levi-Civita connection $\nabla$, it may be given the conharmonic curvature tensor $\widetilde{V}$ on $T^{*} M$ with respect to the metric connection $\widetilde{\nabla}$ as follows:

$\widetilde{V}_{\alpha \beta \gamma \varepsilon}=\widetilde{R}_{\alpha \beta \gamma \varepsilon}-\frac{1}{2(n-1)}\left[\widetilde{R}_{\beta \gamma}\left(\bar{g}_{\nabla, c}\right)_{\alpha \varepsilon}-\widetilde{R}_{\alpha \gamma}\left(\bar{g}_{\nabla, c}\right)_{\beta \varepsilon}-\widetilde{R}_{\beta \varepsilon}\left(\bar{g}_{\nabla, c}\right)_{\alpha \gamma}+\widetilde{R}_{\alpha \varepsilon}\left(\bar{g}_{\nabla, c}\right)_{\beta \gamma}\right]$.

Next we prove the following theorem:

Theorem 7. Let $\nabla$ be a torsion-free linear connection on $M$ and $T^{*} M$ be the cotangent bundle with the modified Riemannian extension $\widetilde{g}_{\nabla, c}$ over $(M, \nabla)$. The cotangent bundle $T^{*} M$ is locally conharmonically flat with respect to the metric 
connection $\widetilde{\nabla}$ if and only if the base manifold $M$ is Ricci flat with respect to $\nabla$ and the components $c_{i j}$ of $c$ satisfy the condition

$$
\nabla_{i}\left(\nabla_{k} c_{j h}-\nabla_{h} c_{j k}\right)-\nabla_{j}\left(\nabla_{k} c_{i h}-\nabla_{h} c_{i k}\right)+R_{i j k}{ }^{m} c_{m h}-R_{i j h}{ }^{m} c_{k m}=0,
$$

where $R_{i j k}{ }^{m}$ denote the components of the curvature tensor $R$ of $\nabla$.

Proof. If the components of the curvature tensor $\widetilde{R}$ of the metric connection $\widetilde{\nabla}$ on $T^{*} M$ satisfy the following equations:

$$
\widetilde{R}_{\alpha \beta \gamma \varepsilon}=\frac{1}{2(n-1)}\left[\widetilde{R}_{\beta \gamma}\left(\bar{g}_{\nabla, c}\right)_{\alpha \varepsilon}-\widetilde{R}_{\alpha \gamma}\left(\bar{g}_{\nabla, c}\right)_{\beta \varepsilon}-\widetilde{R}_{\beta \varepsilon}\left(\bar{g}_{\nabla, c}\right)_{\alpha \gamma}+\widetilde{R}_{\alpha \varepsilon}\left(\bar{g}_{\nabla, c}\right)_{\beta \gamma}\right],
$$

then $T^{*} M$ is said to be locally conharmonically flat with respect to the metric connection $\widetilde{\nabla}$.

On lowering the upper index in the proposition 4, we obtain the components of the $(0,4)$-curvature tensor of the metric connection $\widetilde{\nabla}$ as follows:

$$
\left\{\begin{array}{c}
\widetilde{R}_{i j k l}=+\frac{1}{2}\left\{\nabla_{i}\left(\nabla_{k} c_{j l}-\nabla_{l} c_{j k}\right)-\nabla_{j}\left(\nabla_{k} c_{i l}-\nabla_{l} c_{i k}\right)\right. \\
\left.+R_{i j k}{ }^{m} c_{m l}-R_{i j l}{ }^{m} c_{k m}\right\} \\
\widetilde{R}_{i j k \bar{l}}=R_{i j k}{ }^{l} \\
\widetilde{R}_{i j \bar{k} l}=R_{j i l}{ }^{k} \\
\text { otherwise }=0 .
\end{array}\right.
$$

Putting the values of $\widetilde{R}_{\alpha \beta \gamma \varepsilon}, \widetilde{R}_{\alpha \beta}$ and $\left(\bar{g}_{\nabla, c}\right)_{\beta \varepsilon}$ respectively in (3.20a), we have

$$
\begin{gathered}
\nabla_{i}\left(\nabla_{k} c_{j l}-\nabla_{l} c_{j k}\right)-\nabla_{j}\left(\nabla_{k} c_{i l}-\nabla_{l} c_{i k}\right)+R_{i j k}{ }^{m} c_{m l}-R_{i j l}{ }^{m} c_{k m} \\
=\frac{1}{2(n-1)}\left(R_{j k} c_{i l}-R_{i k} c_{j l}-R_{j l} c_{i k}+R_{i l} c_{j k}\right) \\
R_{i j k}{ }^{l}=\frac{1}{2(n-1)}\left(R_{j k} \delta_{i}^{l}-R_{i k} \delta_{j}^{l}\right) \\
-R_{j i l}{ }^{k}=\frac{1}{2(n-1)}\left(R_{i l} \delta_{j}^{k}-R_{j l} \delta_{i}^{k}\right) .
\end{gathered}
$$

Contraction $i$ and $l$ in (3.22) gives

$$
\begin{aligned}
R_{l j k}^{l} & =\frac{1}{2(n-1)}\left(R_{j k} \delta_{l}^{l}-R_{l k} \delta_{j}^{l}\right) \\
R_{j k} & =\frac{1}{2(n-1)}\left(n R_{j k}-R_{j k}\right) \\
R_{j k} & =\frac{1}{2(n-1)} R_{j k}(n-1) \\
R_{j k} & =0
\end{aligned}
$$

that is, the torsion-free linear connection $\nabla$ is Ricci flat. In the case, from (3.21), it follows that

$$
\nabla_{i}\left(\nabla_{k} c_{j l}-\nabla_{l} c_{j k}\right)-\nabla_{j}\left(\nabla_{k} c_{i l}-\nabla_{l} c_{i k}\right)+R_{i j k}{ }^{m} c_{m l}-R_{i j l}{ }^{m} c_{k m}=0 .
$$




\section{The Schouten-van Kampen connection associated to the Levi-Civita COnNection of the modified Riemannian EXtension}

The Schouten-Van Kampen connection has been introduced in [17] for a study of non-holonomic manifolds. The Schouten-Van Kampen connection associated to the Levi-Civita connection $\bar{\nabla}$ of the modified Riemannian extension $\bar{g}_{\nabla, c}$ and adapted to the pair of distributions $(H, V)$ are defined by

$$
\bar{\nabla}^{*} \widetilde{Y}=H\left(\bar{\nabla}_{\tilde{X}} H \tilde{Y}\right)+V\left(\bar{\nabla}_{\tilde{X}} V \tilde{Y}\right)
$$

for all vector fields $\widetilde{X}$ and $\tilde{Y}$, where $V$ and $H$ are the projection morphism of $T T^{*} M$ on $V T^{*} M$ and $H T^{*} M$ respectively. The formula (4.1) for $\bar{\nabla}^{*}$ has been first given by Ianus (see, [1]). By using (4.1) and (3.3), the Schouten-Van Kampen connection associated to the Levi-Civita connection $\bar{\nabla}$ of the modified Riemannian extension $\bar{g}_{\nabla, c}$ are locally given by the following formulas:

$$
\left\{\begin{array}{c}
\bar{\nabla}_{E_{\bar{i}}}^{*} E_{\bar{j}}=0, \bar{\nabla}_{E_{\bar{z}}}^{*} E_{j}=0, \\
\bar{\nabla}_{E_{i}}^{*} E_{\bar{j}}=-\Gamma_{i h}^{j} E_{\bar{h}}, \bar{\nabla}_{E_{i}}^{*} E_{j}=\Gamma_{i j}^{h} E_{h},
\end{array}\right.
$$

which are the components of the horizontal lift ${ }^{H} \nabla$ of the torsion-free linear connection $\nabla$. Hence we get:

Proposition 5. Let $\nabla$ be a torsion-free linear connection on $M$ and $T^{*} M$ be the cotangent bundle with the modified Riemannian extension $\bar{g}_{\nabla, c}$ over $(M, \nabla)$. The Schouten-Van Kampen connection $\bar{\nabla}^{*}$ associated to the Levi-Civita connection $\bar{\nabla}$ of the modified Riemannian extension $\bar{g}_{\nabla, c}$ and the horizontal lift ${ }^{H} \nabla$ of the torsionfree linear connection $\nabla$ to $T^{*} M$ coincide to each other.

In view of Proposition 3, Proposition 5, Theorem 2 and its proof, it immediately follows the final result.

Theorem 8. Let $\nabla$ be a torsion-free linear connection on $M$ and $T^{*} M$ be the cotangent bundle with the modified Riemannian extension $\bar{g}_{\nabla, c}$ over $(M, \nabla)$. The cotangent bundle $T^{*} M$ is semi-symmetric with respect to the Schouten-Van Kampen connection $\bar{\nabla}^{*}$ associated to the Levi-Civita connection $\bar{\nabla}$ of the modified Riemann-

ian extension $\bar{g}_{\nabla, c}$ if and only if the base manifold $M$ is semi-symmetric with respect to $\nabla$.

\section{REFERENCES}

[1] S. Aslanci, R. Cakan, On a cotangent bundle with deformed Riemannian extension. Mediterr. J. Math. 11 (2014), no. 4, 1251-1260.

[2] Z. Afifi, Riemann extensions of affine connected spaces. Quart. J. Math., Oxford Ser. (2) $5(1954), 312-320$.

[3] E. Calvino-Louzao, E. García-Río, P. Gilkey and A. Vazquez-Lorenzo, The geometry of modified Riemannian extensions. Proc. R. Soc. Lond. Ser. A Math. Phys. Eng. Sci. 465(2009), no. 2107, 2023-2040.

[4] E. Calviño-Louzao, E. García-Río and R. Vázquez-Lorenzo, Riemann extensions of torsionfree connections with degenerate Ricci tensor. Can. J. Math. 62(2010), no. 5, 1037-1057.

[5] A. Derdzinski, Connections with skew-symmetric Ricci tensor on surfaces. Results Math. 52(2008), no. 3-4, 223-245.

[6] V. Dryuma, The Riemann extensions in theory of differential equations and their applications. Mat.Fiz. Anal. Geom. 10(2003), no. 3, 307-325.

[7] E. Garcia-Rio, D. N. Kupeli, M. E. Vazquez-Abal, and R. Vazquez-Lorenzo, Affine Osserman connections and their Riemann extensions. Differential Geom. Appl. 11(1999), no. 2, 145-153. 
[8] A. Gezer, L. Bilen, and A. Cakmak, Properties of modified Riemannian extensions. Zh. Mat. Fiz. Anal. Geom. 11 (2015), no. 2, 159-173.

[9] H. A. Hayden, Sub-spaces of a space with torsion. Proc. London Math. Soc. S2-34 (1932), 27-50.

[10] T. Ikawa, K. Honda, On Riemann extension. Tensor (N.S.) 60 (1998), no. 2, 208-212.

[11] S. Ianus, Some almost product structures on manifolds with linear connection. Kodai Math. Sem. Rep. 23(1971), 305-310.

[12] Y. Ishii, On conharmonic transformations. Tensor 7 (1957), no. 2, 73-80.

[13] O. Kowalski and M. Sekizawa, On natural Riemann extensions. Publ. Math. Debrecen 78(2011), no. 3-4, 709-721.

[14] C. A. Mantica and L. G. Molinari, Weakly Z symmetric manifolds. Acta Math. Hungar. 135 (2012), no.1-2, 80-96 .

[15] K. P. Mok, Metrics and connections on the cotangent bundle. Kodai Math. Sem. Rep. 28 (1976/77), no. 2-3, 226-238.

[16] E. M. Patterson and A. G.Walker, Riemann extensions. Quart. J. Math. Oxford Ser. (2) $3(1952), 19-28$.

[17] J. A. Schouten and E. R. van Kampen, Zur Einbettungs- und Krummungstheorie nichtholonomer Gebilde. Math. Ann. 103(1)(1930), 752-783.

[18] N. S. Sinjukov, Geodesic mappings of Riemannian spaces (Russian). Publishing House "Nauka", Moscow, 1979.

[19] Z. I. Szabo, Structure theorems on Riemannian spaces satisfying $R(X, Y) \cdot R=0$. I. The local version, J. Differential Geom. 17 (1982), 531-582.

[20] Z. I. Szabo, Structure theorems on Riemannian spaces satisfying $R(X, Y) \cdot R=0$. II. Global version, Geom. Dedicata 19 (1985), 65-108.

[21] M. Toomanian, Riemann extensions and complete lifts of s-spaces. Ph. D. Thesis, The university, Southampton, 1975.

[22] L. Vanhecke and T. J. Willmore, Riemann extensions of D'Atri spaces. Tensor (N.S.) 38(1982), 154-158.

[23] T. J.Willmore, Riemann extensions and affine differential geometry. Results Math. 13(1988), no. 3-4, 403-408.

[24] K. Yano, S. Ishihara, Tangent and Cotangent Bundles. Marcel Dekker, Inc., New York 1973.

Igdir University, Faculty of Science and Letters, Department of Mathematics and COMPuTER, 76000, IGdir-Turkey.

E-mail address: lokman.bilen@igdir.edu.tr

Ataturk University, Faculty of Science, Department of Mathematics, 25240, ErzurumTURKEY.

E-mail address: agezer@atauni.edu.tr 